\title{
Distinct roles of prostaglandin $H$ synthases 1 and 2 in T-cell development
}

\author{
Bianca Rocca, ${ }^{1}$ Lisa M. Spain, ${ }^{2}$ Ellen Puré, ${ }^{2}$ Robert Langenbach, ${ }^{3}$ Carlo Patrono, ${ }^{4}$ \\ and Garret A. FitzGerald ${ }^{1}$
}

${ }^{1}$ Center for Experimental Therapeutics, University of Pennsylvania, Philadelphia, Pennsylvania 19104, USA
${ }^{2}$ The Wistar Institute, Philadelphia, Pennsylvania 19104, USA
${ }^{3}$ National Institute of Environmental Health Sciences, Laboratory of Experimental Carcinogenesis and Mutagenesis,
Research Triangle Park, North Carolina 27709, USA
${ }^{4}$ Department of Medicine and Aging, University of Chieti “G. D’Annunzio” School of Medicine, Chieti, Italy

Address correspondence to: Garret A. FitzGerald, Center for Experimental Therapeutics, 153 Johnson Pavillion, University of Pennsylvania, Philadelphia, Pennsylvania 19104, USA. Phone: (215) 898-1184; Fax: (215) 573-9135;

E-mail: garret@spirit.gcrc.upenn.edu.

Received for publication January 28, 1999, and accepted in revised form April 16, 1999.

Prostaglandin $\mathrm{G}$ and $\mathrm{H}$ synthases, or cyclooxygenases (COXs), catalyze the formation of prostaglandins (PGs). Whereas COX-1 is diffusely expressed in lymphoid cells in embryonic day 15.5 thymus, COX-2 expression is sparse, apparently limited to stromal cells. By contrast, COX-2 is predominant in a subset of medullary stromal cells in three- to five-week-old mice. The isozymes also differ in their contributions to lymphocyte development. Thus, experiments with selective COX-1 inhibitors in thymic lobes from normal and recombinase-activating gene- 1 knockout mice support a role for this isoform in the transition from $\mathrm{CD}^{-} \mathrm{CD}^{-}$- double-negative (DN) to $\mathrm{CD} 4^{+} \mathrm{CD}^{+}$double-positive (DP). Concordant data were obtained in COX-1 knockouts. Pharmacological inhibition and genetic deletion of COX-2, by contrast, support its role during early thymocyte proliferation and differentiation and, later, during maturation of the CD4 helper T-cell lineage. $\mathrm{PGE}_{2}$, but not other PGs, can rescue the effects of inhibition of either isoform, although it acts through distinct EP receptor subtypes. COX-dependent PG generation may represent a mechanism of thymic stromal support for T-cell development.

J. Clin. Invest. 103:1469-1477 (1999).

\section{Introduction}

Prostaglandins (PGs) are bioactive lipids formed by the sequential actions of cyclooxygenase- 1 and -2 (COX-1 and COX-2) and specific PG synthases (1). The known functions of the largely constitutive enzyme, COX-1, include generation of proaggregatory TxA2 by platelets, production of gastroprotective PGs, and regulation of water and salt reabsorption in the kidney (1). In contrast, COX-2 expression is induced in macrophages, fibroblasts, vascular endothelial cells and smooth muscle cells by shear stress, cytokines, and growth factors and accounts for PG formation during inflammatory responses, reproduction, and renal adaptation to systemic stress (2).

PGs have been shown to regulate immune responses mediated by mature B and T lymphocytes. Prostaglandin $\mathrm{E}_{2}\left(\mathrm{PGE}_{2}\right)$ shifts the balance within the $\mathrm{T}$ lineage of the cellular immune response away from T-helper type 1 cells toward T-helper type 2 cells by inhibiting IL-2 and enhancing IL-4 production (3-8). PGE $_{2}$ directly regulates the activation of mature $B$ lymphocytes by skewing their differentiation toward IgE production (9). An immunoregulatory role for $\mathrm{PGE}_{2}$ is also suggested by its overproduction, either in vivo or ex vivo, in disorders that feature impaired immunological responses, including AIDS $(10,11)$, bone marrow or stem cell transplantation (12), atopic dermatitis, and the hyper-IgE syndrome (13). Several observations implicate PGs in the maturation of the T-cell lineage. Expression of various PG biosyn- thetic enzymes and receptors has been detected in the thymus (14-17). Furthermore, thymus and nonlymphoid thymic stromal cell lines have been shown to secrete PGs in vitro (18-20). We now report that expression of the COX isoforms in mouse thymus is spatially and temporally distinct. Moreover, the products of these isozymes subserve distinct roles at critical stages in T-cell maturation. COX inhibitors may act, in part, by modulating immune function.

\section{Methods}

Mice. C57Bl/6J wild-type and recombinase-activating gene1-deficient mice $\left(R A G-1^{-/}\right)$were purchased from The Jackson Laboratory (Bar Harbor, Maine, USA); COX-1- and COX2 -deficient mice were generated as described previously (21, 22). Pregnancies were timed from the first day of plug observation (day 0.5).

Fetal thymic organ cultures. Fetal thymic organ cultures (FTOCs) were performed according to procedures described previously (23). Cultures were fed daily by complete medium replacement with solvent control or the following drugs (in DMSO or ethanol): indomethacin (Sigma Chemical Co., St. Louis, Missouri, USA), ibuprofen (Sigma Chemical Co.), valeryl salicylate (Cayman Chemical, Ann Arbor, Michigan, USA), prostanoids (Cayman Chemical) or prostanoid analogues (Cayman Chemical; Bayer AG, Leverkusen, Germany), NS-398 (Biomol Research Laboratories, Plymouth Meeting, Pennsylvania, USA), L759,700, and L-745,337 (kindly provided by W. Tanaka, Merck Research Laboratories, Rahway, New Jersey, USA.)

Flow cytometric analyses. Thymic lobes were mechanically dissociated and stained using standard procedures. The following 
mAb's were used: anti-CD4 FITC, anti-CD8 Red 613, and antiCD3e phycoerythrin (GIBCO BRL, Gaithersburg, Maryland, USA); or anti-CD4 FITC, anti-CD8 FITC (GIBCO BRL), antiCD25 phycoerythrin (PharMingen, San Diego, California, USA), biotinylated CD44, and streptavidin Red 670 (GIBCO $B R L)$. Flow cytometric analysis was performed on a FACScan (Becton Dickinson Immunocytometry Systems, San Jose, California, USA) and analyzed using CellQuest software (Becton Dickinson). Aliquots from each cell suspension were removed to determine the total cell number and viability. The relative percentages of various thymocyte subpopulations based on CD4 and CD8 expression were obtained using quadrant statistics in CellQuest. The absolute numbers of each thymocyte subset were calculated by multiplying the total cell number by the percentage of each thymocyte population. The purity of flowsorted populations of thymocytes was $>99 \%$.

RT-PCR analyses. Total RNA was extracted with an RNeasy Mini Kit (QIAGEN Inc., Valencia, California, USA). RT-PCR analyses were performed using standard procedures with primers specific for COX-1 (sense: 5'-GGTCCTGCTCGCAGATC-CTG-3'; antisense: 5'-CCTCTGGAAAGATGGGTCCT$3^{\prime}$ ), COX-2 (sense: 5'-CTGTACAAGCA-GTGGCAA-3'; antisense: 5'-TTACAGCTCAGTTGAACGCCT-3'), and the prostaglandin EP4 receptor (24) (sense: 5'-AGCGCCTCAGCGACTTTCGCC-3'; antisense: 5'-AGGCACTTGATCTTCTCTATG-3'). The mRNA amplifications of other EPs were performed as described (25). PCR products were confirmed by Southern blotting; the filters were hybridized using specific oligonucleotides (COX-1: 5'-TTCTT-TGCACAACACTTCA3'; COX-2: 5'-CATTAACCCTA-CAGTACTAAT-3'; EP1: 5'GCTTTGGCAG-TGGCGCTGTGCTGCCA-3'; EP2: 5'TAATTGAC-CCTTGGGTCTTTGCCA-3'; EP3 isoforms:
5'-ACACAAACTAATGCTTCCAGCTCCA-3'; EP4: 5'ATCGCCACCTCTCTGGTGGTG-3') after $5^{\prime}$ end labeling with $\left[{ }^{32} \mathrm{P}\right]$ ATP.

Immunohistochemistry. Thymic lobes were fixed with sucrose, embedded in OCT compound, and frozen and stained using standard procedures. The primary antibodies were COX-1 antiserum (1:100-1:200 dilution), COX-2 antiserum (1:200-1:400 dilution) (kindly provided by A. Habib and the late J. Maclouf, INSERM, Paris, France; 26), anti-Thy $1.2(20 \mu \mathrm{g} / \mathrm{mL}) \mathrm{mAb}$, anti-MHC class II $(20 \mu \mathrm{g} / \mathrm{mL}) \mathrm{mAb}(27)$, ulex europaeus agglutinin-1 (UEA-1) lectin (Vector Laboratories, Burlingame, California, USA) (1:100 dilution), anti-6C3 (culture supernatant, undiluted), anti-cytokeratin $8.13 \mathrm{mAb}$ (Sigma Chemical Co.) (1:100 dilution), or anti-CDR1 mAb (1:10 dilution).

Western blot analysis. Thymi were washed twice with PBS buffer and lysed for Western blotting using standard procedures. Membranes were incubated with specific mAb's as described previously (26). Chemiluminescent substrates (ECL; Amersham Life Sciences Inc., Arlington Heights, Illinois, USA) were used according to the manufacturer's instructions, and bands were observed after a fixed time exposure (1-2 minutes) to Hyperfilm-ECL (Amersham Life Sciences Inc.). Protein bands were quantified using an LKB Ultrascan XL laser densitometer (Amersham Pharmacia Biotech, Piscataway, New Jersey, USA). Immunodetection was linear between 15 and $75 \mu \mathrm{g}$ of COX-1 and COX-2.

Statistical analysis. The results were evaluated by using ANOVA, with subsequent comparisons using Student's $t$ test for paired or nonpaired data as appropriate. Statistical significance was defined as $P<0.05$. Values were reported as the mean $\pm 1 \mathrm{SD}$. The $\mathrm{IC}_{50}$ was calculated using Biosoft-Dose software (ElsevierBiosoft, Cambridge, United Kingdom).

\section{Figure 1}

Characterization of COX-1 and COX2 mRNA and protein expression. (a) Total RNA from indicated tissues or fractions was isolated, and cDNAs were amplified by RT-PCR using primers specific for COX-1 (left), COX-2 (right), or actin (see Methods). The identity of the amplified fragments for COX-1 or COX-2 was confirmed by Southern blot analysis with specific probes. -, negative control; +, positive control (NIH $3 \mathrm{~T} 3$ cells); DN, CD4-CD8- thymocytes; $\mathrm{DP}, \mathrm{CD}^{+} \mathrm{CD}^{+}$thymocytes; 4SP, CD4+ lymphocytes; E15.5, embryonic day 15.5 thymus; FTOC, E15.5 thymus cultured for 5 days. (b) Frozen sections of E15.5 thymus were reacted with normal rabbit IgG as a negative control (panel 1), anti-COX-1 (panel 2), anti-COX-2 (panel 3), and anti-Thy 1.2 (panel 4). Sections were counterstained with hematoxylin. $\times 40$. (c) Frozen sections of thymus from 3-week-old mice were double stained with UEA-1 lectin (left) and anti-COX-2 (right). C, cortex; M, medulla. $\times 100$. a
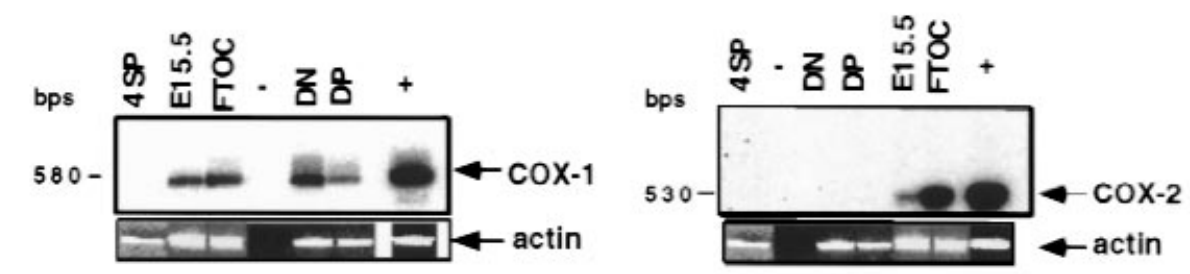

b
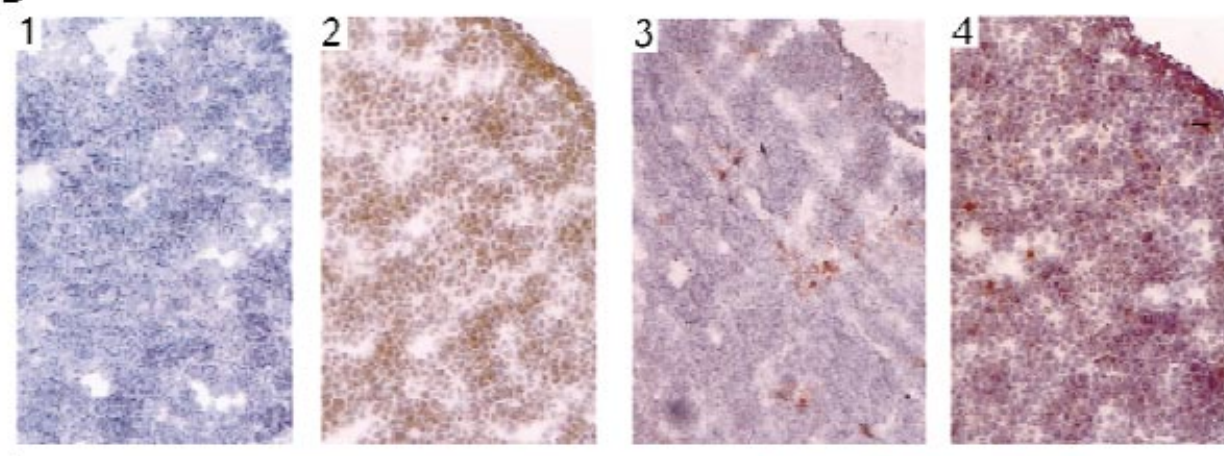

c

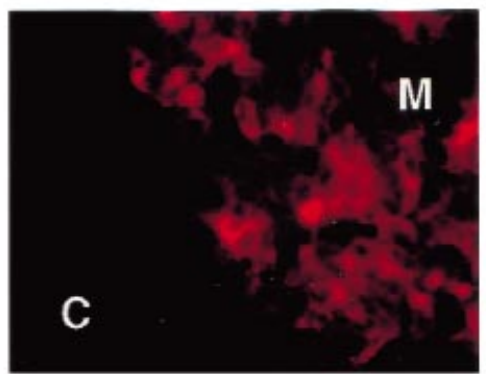




\section{Results}

Expression of COX-1 and COX-2 in thymi and isolated thymocytes. COX-1 and COX-2 products of the expected size were amplified by RT-PCR from total RNA of embryonic day 15.5 (E15.5) thymi, E15.5 cultured thymic lobes, and different thymocyte subpopulations purified by cell sorting, based on CD4 and CD8 expression. COX-1 and COX-2 products of the expected size were amplified from total RNA of E15.5 thymi and from E15.5 FTOCs (Figure 1a). A specific product for COX-1 was amplified from RNA of both CD4-CD8- double-negative (DN) and $\mathrm{CD}^{+} \mathrm{CD}^{+}$double-positive (DP) thymocytes, but not from $\mathrm{CD}^{+}$single-positive (SP) mature lymphocytes (Figure 1a). COX-2 transcript was not detectable in purified DN, DP, or CD4 ${ }^{+}$SP cells (Figure 1a).

Sections of E15.5 thymi were immunostained for COX-1 or COX-2 proteins, for the Thy 1.2 antigen, or for the MHC class II molecule. COX-1 staining showed a diffuse pattern of expression in E15.5 thymi similar to that in Thy 1.2 (Figure 1b, panels 2 and 4). The majority of cells positive for COX-1 had lymphoid morphology at higher power $(\times 40-100)$ (data not shown). Fewer, sparsely distributed cells expressed COX-2 in E15.5 thymus (Figure 1b, panel 3). They demonstrated stromal cell morphology at higher power and were MHC class II positive (data not shown). We also investigated COX-2 expression in the adult (3- to 5-week-old) thymus to characterize further the stromal component expressing COX-2. Thymic sections were stained for COX-2, medulla-specific UEA-1 lectin, cytokeratin, 6C3, or CDR1 (cortex-specific antibodies). The majority of the COX-2-positive cells were positive for UEA-1 and cytokeratin (Figure $1 \mathrm{c}$ and data not shown) and were localized near the corticomedullary junction (Figure 1c). Thus, the COX isoforms are differentially expressed in the thymus, and COX-2 is constitutively expressed in thymic medullary stroma.

$C O X-1$ is required for $C D 4^{+} C D 8^{+}$thymocyte formation. We next investigated T-cell development in the absence of COX activity. We used selective COX inhibitors in FTOCs and analyzed CD4, CD8, and CD3e expression. More than $90 \%$ of thymocytes in normal mice lack CD4, $\mathrm{CD} 8$, and $\mathrm{CD} 3 \varepsilon$ expression at E15.5, whereas by E17-18.5, the majority of thymocytes (70-80\%) have progressed to the DP stage. After E17.5, CD4 and CD8 SP $\mathrm{CD}^{+}$thymocytes develop from DP intermediates. This developmental progression is recapitulated in FTOC with a minimal lag $(23,27)$.

Two highly selective COX-1 inhibitors were used: valeryl salicylate (28) and the more potent L-759,700, which has roughly a 100 -fold preference for COX-1 over COX-2 in different cellular systems (ref. 29, and unpublished data) Both inhibitors were provided from Merck Research Laboratories. We also used ibuprofen and indomethacin as nonselective COX-1 and COX-2 inhibitors $(30,31)$. All inhibitors caused dose-dependent, statistically significant increases of 4- to 5-fold in the percentage of DN cells in E15.5 FTOCs, whereas the DP subset was decreased concomitantly (Figure 2a). A dose-dependent increase in the number of DN cells was also observed in the presence of the inhibitors. The $\mathrm{IC}_{50}$ values for the inhibition of $\mathrm{DP}$ absolute counts were as follows L-759,700, $0.7 \mu \mathrm{M}$;

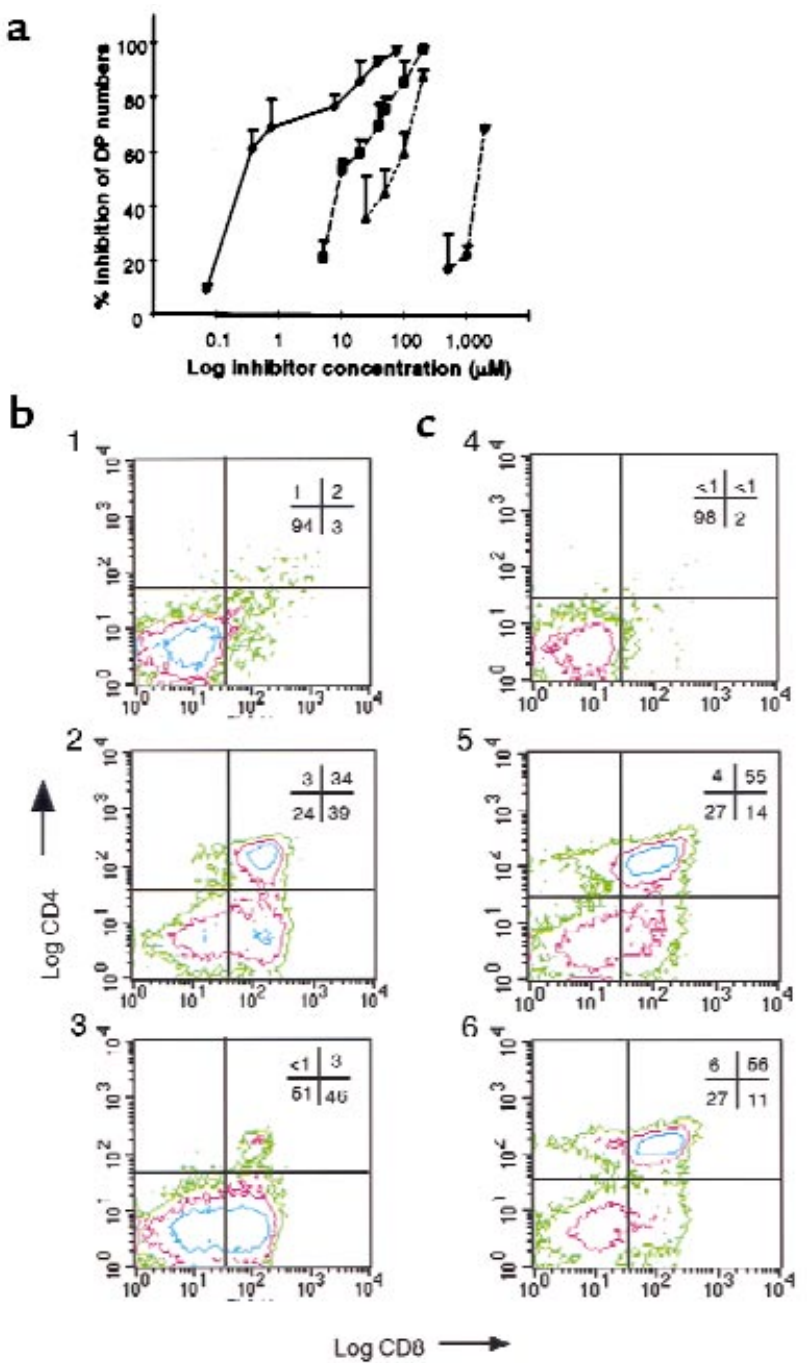

Figure 2

Effect of COX-1 inhibition on DP formation. (a) E15.5 FTOCs were treated with different concentrations of L-759,700 (filled diamonds), indomethacin (filled squares), ibuprofen (filled triangles), or valeryl salicylate (open circles) for 5 days of culture. The percent inhibition of the DP absolute counts is expressed relative to vehicle-treated FTOCs. Values are means \pm SD of 3-6 different experiments, each performed in quadruplicate. (b) Effect of the selective COX-1 inhibitor L-759,700 on E15.5 RAG-1-1- FTOCs. The panels show a representative experiment in which $R A G-1^{-1-}$ thymic lobes were cultured in the presence of vehicle (panel 1),

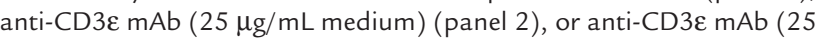
$\mu \mathrm{g} / \mathrm{mL}$ medium) plus L-759,700 (7 $\mu \mathrm{M})$ (panel 3). (c) Effect of the selective COX-2 inhibitor NS-398 on E15.5 RAG-1-/- FTOCs. RAG-1-/- thymic lobes were cultured for 5 days and analyzed for CD4 and CD8 expression by flow cytometry. Conditions were as follows: vehicle (panel 4), antiCD3 $\varepsilon \mathrm{mAb}(25 \mu \mathrm{g} / \mathrm{mL}$ medium) (panel 5), or anti-CD3ع mAb $(25 \mu \mathrm{g} / \mathrm{mL}$ medium) plus NS-398 (40 $\mu \mathrm{M})$ (panel 6).

indomethacin, $9 \mu \mathrm{M}$; ibuprofen, $53 \mu \mathrm{M}$; and valeryl salicylate, $1.5 \mathrm{mM}$ (Figure 2a). These $\mathrm{IC}_{50}$ were similar to the $\mathrm{EC}_{50}$ observed for the increase in the percentage of $\mathrm{DN}$ cells. The relative potency of these 4 compounds in FTOCs corresponded to their rank order of potency for inhibition of COX-1 in different systems (30) (Table 1). These effects were reversed by selected PG analogues (see below). Forward- and side-light measurements, as well as propidium iodide staining for fragmented DNA, showed no increase 


\section{Figure 3}

Flow cytometry analysis of thymocytes from E17.5 and E18.5 FTOCs treated with selective COX-1 inhibitor. Thymocytes from E17.5 plus 5 days culture in FTOCs were examined for CD4 and CD8 expression. (a) Vehicle. (b) L-759,700, $10 \mu \mathrm{M}$. The percentage of cells in each quadrant are indicated in the corners of each graph. (c) E15.5 (filled circles), E17.5 (filled squares), and E18.5 (filled triangles) FTOCs were treated with different concentrations of L759,700 . The percent inhibition of the DP absolute counts is expressed relative to vehicle-treated FTOCs. Values are means \pm SD of 3-6 different experiments, each performed in quadruplicate.
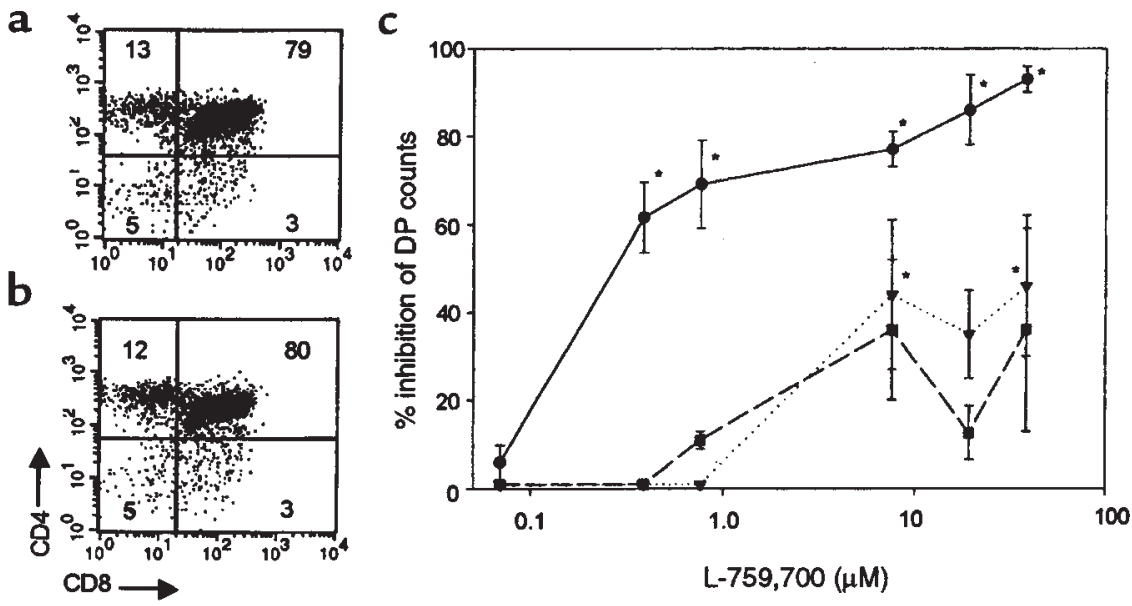

in apoptotic thymocytes in E15.5 inhibitor-treated FTOCs (data not shown).

We examined mice with a targeted disruption of the recombinase-activating gene-1 (RAG-1 $\left.1^{-/}\right)$to clarify further the role of COXs in lymphocyte maturation. Thymocyte development in $R A G-1^{-/-}$animals is arrested at the $\mathrm{DN}$ stage in vivo because of a failure to rearrange the T-cell receptor (TCR) $\beta$ locus (32). Incubation of $R A G-1^{-1-}$ thymic lobes with anti-CD $3 \varepsilon$ mAb in FTOCs results in efficient progression of the DN thymocytes to the DP stage (33). DPs were formed in E15.5 RAG-1 $1^{-/}$FTOCs treated with anti-CD $3 \varepsilon$ mAb alone (Figure $2 b$, panels 1 and 2). However, when lobes were coincubated with anti$\mathrm{CD} 3 \varepsilon \mathrm{mAb}$ and the COX-1-specific inhibitor L-759,700, the development of DP thymocytes was greatly reduced (Figure 2b, panel 3). The selective COX-2 inhibitor NS398 (34) at concentrations up to $80 \mu \mathrm{M}$ did not affect the formation of DP thymocytes under the same experimental conditions (Figure 2c). The number of cells recovered in each case was also determined. In the experiments shown in Figure 2, approximately $15 \times 10^{4}$ cells per lobe were recovered from untreated samples. In the presence of anti-CD $3 \varepsilon$, cell recovery increased to $76 \times 10^{4}$ cells per lobe. The DN-to-DP transition has been shown previously to be associated with expansion of thymocyte numbers (33). The addition of L-759,700 reduced recovery to $38 \times 10^{4}$ cells per lobe, which is consistent with a developmental block in the DN-to-DP transition. NS-398 also reduced cell numbers by approximately 2 -fold in the absence of any change in subset frequency (Figure 2c). This reduction is likely due to an effect of the inhibitor on an earlier stage of thymocyte proliferation (see below).

We also considered the possibility that the COX-1 inhibitors could reduce the survival of DP thymocytes, thereby decreasing their numbers and increasing the frequency of DN thymocytes. To test this possibility, we allowed fetal development to proceed in vivo to E17.5 and 18.5, and then initiated 5-day FTOCs in the presence or absence of L-759,700. By E17.5, DP thymocytes are already formed in vivo, and they transit to the CD4 SP mature stage during 5 days of organ culture (23). L-759,700 was much less effective in reducing the percentages and absolute numbers of the DP thymocytes in E17.5 and 18.5 relative to E15.5 thymi (Figure 3, a and b). Some reductions in DP cell numbers were observed at higher doses (Figure 3c). These reductions could reflect an inhibitormediated block of the remaining DN cells in the older thymi undergoing the DP transition during the culture period. In addition, L-759,700 did not affect the percentage or number of CD4 or CD8 SP CD3 $\varepsilon^{+}$thymocytes (Figure 3). Taken together, these observations support the hypothesis that the COX-1 inhibitor interferes with the DN-to-DP transition by blocking either differentiation, proliferation, or survival of differentiating cells. Our data argue against the possibility that the COX-1 inhibitor is directly toxic to preformed DP thymocytes.

We confirmed these results in COX-1-deficient mice $\left(\mathrm{COX}-1^{-/-}\right)$. E15.5 thymi isolated from $\mathrm{COX}-1^{-/-}$and wildtype embryos showed no differences in the total cell number or the phenotype of thymocytes (>90\% were in the DN stage; data not shown). However, by E17.5, a significantly higher percentage of DN and lower percentage of DP thymocytes were consistently observed in E17.5 $\mathrm{COX}-1^{-/-}$ embryos compared with wild-type embryos (Figure 4a). Therefore, we investigated the DN-to-DP transition by culturing E15.5 thymi. Again, thymi from $\mathrm{COX}-1^{-/-}$mice showed a significantly $(P<0.001)$ reduced percentage and number of DP cells, with a concomitant increase in DN

\section{Table 1}

Relative concentration of COX inhibitors required for the increase in the percentage of $\mathrm{CD}^{-} \mathrm{CD}^{-}$cells, reduction in $\mathrm{CD}^{+} \mathrm{CD} 8^{+}$absolute numbers and COX-1 inhibition.

\begin{tabular}{lccc}
\hline Inhibitor & $\begin{array}{c}\text { DN } \\
\text { increase }\end{array}$ & $\begin{array}{c}\text { COX-1 } \\
\text { inhibition }\end{array}$ & $\begin{array}{c}\text { DP } \\
\text { reduction }\end{array}$ \\
L-759,700 & $1^{\mathrm{A}}$ & $1^{\mathrm{A}}$ & $1^{\mathrm{A}}$ \\
Indomethacin & 18 & 6 & 13 \\
Ibuprofen & 67 & 84 & 76 \\
Valeryl salicylate & 2,500 & 9,618 & 2,143 \\
\hline
\end{tabular}

${ }^{A}$ The $I^{50}$ s values for L-759,700 are: $0.6 \mu \mathrm{M}$ (DN increase), $0.11 \mu \mathrm{M}$ (COX-1 inhibition), $0.7 \mu \mathrm{M}$ (DP reduction). Standardizing these values at unity, the corresponding values for the other compounds are expressed as multiples. Data for COX1 inhibition by the 4 compounds are derived from measurement of human platelet COX-1 activity in whole blood (30). 
cells. DN percentages were increased 2 -fold in $\mathrm{COX}-1^{-/-}$ mice relative to wild-type littermates $\left(28 \pm 8 \%\right.$ in $\mathrm{COX}^{-1} 1^{-/}$ vs. $12 \pm 3 \%$ in wild-type mice), as were $\mathrm{DN}$ absolute numbers $\left(19 \pm 4 \times 10^{4}\right.$ cells per lobe in COX-1-/- vs. $11 \pm 4 \times 10^{4}$ in wild-type mice). DP percentages were slightly but significantly $(P<0.001)$ decreased in $\mathrm{COX}^{-1} 1^{-/}$mice relative to wild-type littermates $\left(54 \pm 8 \%\right.$ in $C O X-1^{-/-}$vs. $70 \pm 5 \%$ in wild-type), as were absolute numbers ( $40 \pm 16 \times 10^{4}$ cells per lobe in $C O X-1^{-/-}$vs. $58 \pm 14 \times 10^{4}$ in wild-type mice).

Clearly, the requirement for COX-1 is not absolute in the knockout mice because many DP thymocytes are formed, and these thymocytes can continue differentiation to the SP stage. In fact, although the percentage of DP cells was still significantly lower in $\mathrm{COX}-1^{-/-}$compared with wildtype organs in embryonic and adult mice ( 5 weeks, data not shown), a significant increase in both the relative percentage and absolute number of CD4 SP lymphocytes was observed in null thymi (Figures $4 \mathrm{~b}$ and $5 \mathrm{a}$ ). Because our further results (see below) indicate that COX-2 plays a role in CD4 SP formation, we investigated whether increased COX-2 expression might compensate for the absence of COX-1. COX-2 protein was increased approximately 2.4fold in $\mathrm{COX}-1^{-/-}$thymi (Figure $5 \mathrm{~b}$ ).

COX-2 is required at 2 steps of $T$-cell development. We next investigated the role of COX-2 in T-cell formation. The relative distribution of DN vs. DP subsets in E17.5 COX$2^{-/-}$thymi was similar to that in wild-type embryos, indicating that, in contrast to COX-1, COX-2 is not involved in DN-to-DP transition (data not shown and Figure 6a). However, CD4 SP lymphocyte formation was impaired in $\mathrm{COX}-2^{-/-}$E17.5 thymi cultured for 5 days (Figure $6 \mathrm{a}$ ). There was a $50 \%$ reduction in the relative percentage and number of CD4 SP cells in COX-2-/- embryos $(P<0.001)$ (Figure 6a). Although the relative percentage of the DP cells was increased by roughly $10 \%$ in the COX-2 $2^{-/-}$thymi (Figure 6a), their absolute counts were not significantly different from controls. No significant differences in percentage or absolute counts were observed for the CD8 SP subset. Furthermore, the DN counts were reduced by approximately $45 \%$ in E17.5 $\mathrm{COX}-2^{-/-}$cultured thymic lobes compared with controls $(P<0.01)$. COX-1 protein was not upregulated in $C O X-2^{-/-}$thymi (data not shown).

The results with the COX-2-/- mice were confirmed by pharmacological inhibition of COX-2. NS-398 and L745,337 are reportedly 160 - and 245 -fold more potent on average as COX-2 and COX-1 inhibitors, respectively (34). Neither NS-398 nor L-745,337 caused any change in the relative proportions of DN and DP subsets in E15.5 FTOCs. These data and the observation that NS398 did not impair the DP formation in antiCD3e-treated $R A G-1^{-/-}$FTOCs (Figure 2c) confirmed that, in contrast to COX-1, COX-2 is not involved in this transition. However, in E17.5 FTOCs, NS-398 caused a dose-dependent decrease in the percentage and number of CD4 SP cells, with an $\mathrm{IC}_{50}$ of $30 \mu \mathrm{M}$ (Figure $6 \mathrm{~b}$ and data not shown). No such decrease was observed with the specific COX-1 inhibitor L-759,700 (Figure 3). No significant change in CD8 SP thymocytes was observed, even when the cells were gated, based on expression of CD3e (data not shown). In addition, the DN cell counts were also dose dependently reduced by NS-398 (IC 50 of $30 \mu \mathrm{M})$. The DP percentages were slightly increased (Fig-

\section{a $\mathbf{E 1 7 . 5}$}
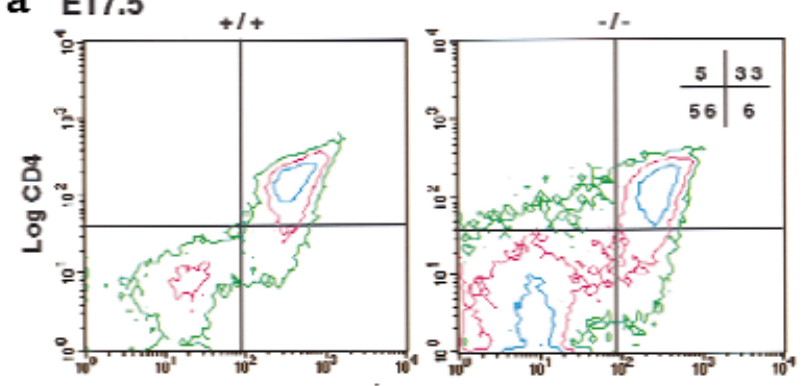

b $E 17.5+5$

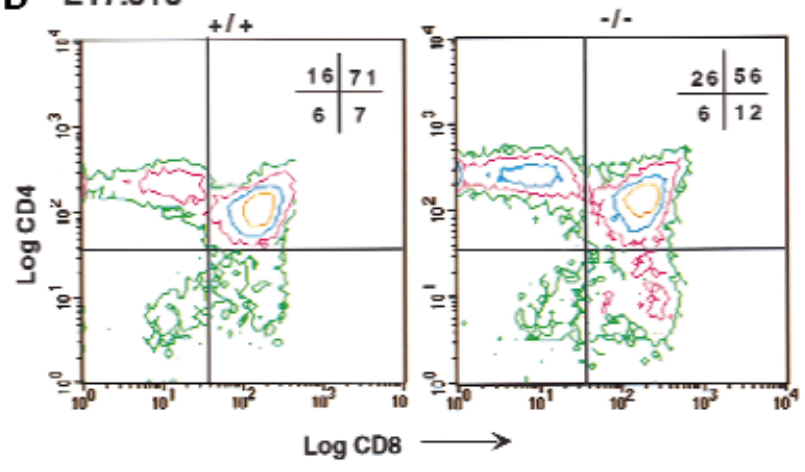

Figure 4

Flow cytometry analysis of thymocytes from E17.5 thymi and from E17.5 cultured $\mathrm{COX}_{-11^{-1-}}$ and control thymi. Thymocytes from $\mathrm{COX}-1^{-1-}$ and control mice were examined for CD4 and CD8 expression. (a) E17.5 thymi. (b) E17.5 thymi in culture 5 days. +/+, controls; -/-, COX-1-/mice. The percentage of cells in each quadrant is indicated in the corners of each graph.

\section{a}

b
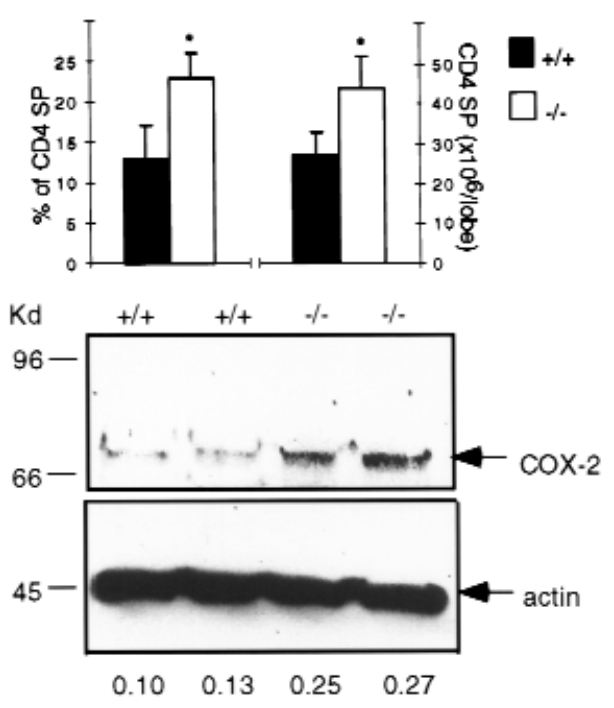

\section{Figure 5}

CD4 SP cells and COX-2 protein levels in COX-1-1- and wild-type thymi. (a) Thymocytes were isolated from adult (5-week-old) thymi, counted, and analyzed by flow cytometry. The percentage and absolute counts of CD4 SP cells are shown in the graphs. ${ }^{*} P<0.01$ vs. controls; $n=4$ each group. (b) Western blot analysis using proteins $(50 \mu \mathrm{g})$ extracted from 5 week-old thymi and using antibodies specific for COX- 2 or $\beta$-actin as controls. The numbers indicate the actin/COX-2 ratios of the densitometric values of the bands. $+/+$, wild-type mice; $-/-, \mathrm{COX}_{-1^{-/-}}$mice. 


\section{a $\quad \mathrm{E} 17.5+5$}

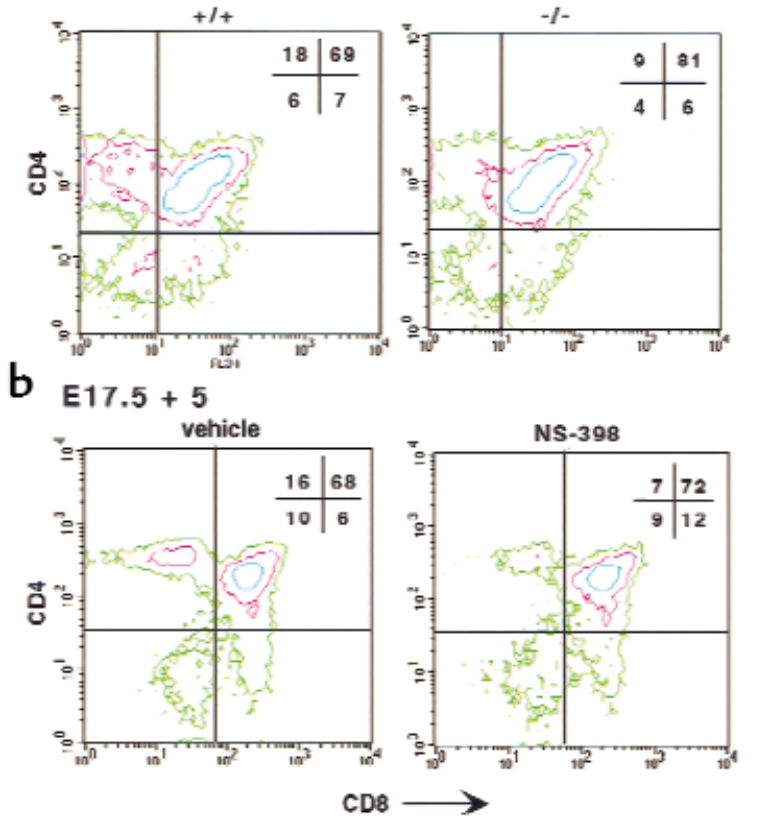

Figure 6

Flow cytometric analyses of thymocytes from COX-2-/- E17.5 FTOCs and wild-type E17.5 FTOCs in the presence of COX-2 inhibitors. (a) E17.5 thymi were isolated from $\mathrm{COX}-2^{-/-}$embryos (-/-) and control siblings $(+/+)$and cultured for 5 days. Thymocytes were analyzed for CD4 and CD8 expression by flow cytometry. The contour plots are shown. Percentages are indicated in the corner of each quadrant. (b) The same experiments and analyses were performed in wild-type E17.5 FTOCs in the presence of the selective COX-2 inhibitor NS-398 (40 $\mu \mathrm{M})$ or vehicle.

ure 6b), but their absolute numbers were not significantly affected. L-745,337 showed effects similar to NS398 in E17.5 FTOCs, with an $\mathrm{IC}_{50}$ of $82 \mu \mathrm{M}$. The relative difference in the $\mathrm{IC}_{50}$ between NS-398 and L-745,337 is consistent with their respective potencies in inhibiting COX-2 in a different cellular system (34).

We further investigated the effects of selective COX-2 inhibition on the different DN thymocyte subsets by staining for CD44 and CD25 antigens (35). NS-398 caused a concentration-dependent decrease in the total cell counts per lobe in E15.5 RAG-1 $1^{-/-}$FTOCs $\left(\mathrm{IC}_{50}\right.$ of 31 $\mu \mathrm{M})$. The percentages and absolute counts of the CD $44^{-} \mathrm{CD} 25^{+}$subset were reduced with the same $\mathrm{IC}_{50}$. On the other hand, the proportions of the 2 most immature subsets of DN thymocytes $\left(\mathrm{CD} 44^{+} \mathrm{CD} 25^{-}\right.$and $\mathrm{CD} 44^{+} \mathrm{CD} 25^{+}$) showed a parallel increase, but their counts were not significantly affected by NS-398 (data not shown). Similar results were obtained with E15.5 wild-type FTOCs. The selective COX-1 inhibitor L759,700 showed no effect on the DN subsets in the same experimental conditions (data not shown). These data are consistent with a role for COX-2 in the maturation of CD4 SP cells and in the survival or further differentiation of thymocytes at the $\mathrm{CD} 44^{-} \mathrm{CD} 25^{+}$stage.

$P G E_{2}$ regulates development by different receptor subtypes. To determine which PGs might be involved in the maturation of DN thymocytes, we rescued the impairment in development by adding back prostanoids or their synthetic analogues to inhibitor-treated FTOCs. E15.5 FTOCs were incubated in the presence of indomethacin $(40 \mu \mathrm{M})$, with increasing concentrations of $\mathrm{PGE}_{2}$ or one of its synthetic analogues: $\mathrm{PGF}_{2 \alpha}, \mathrm{U} 46619$ (a stable $\mathrm{PGH}_{2} / \mathrm{TXA}_{2}$ analogue), iloprost (a stable $\mathrm{PGI}_{2}$ analogue), or 15 -deoxy- $\Delta^{12,14}-\mathrm{PGJ}_{2}$ (a putative metabolite of $\mathrm{PGD}_{2}$ ). $\mathrm{PGE}_{2}$, but none of the other prostanoids, overcame the inhibitory effect of indomethacin in E15.5 FTOCs (Figure $7 \mathrm{a}$ ). In addition, neutralizing antibodies to $\mathrm{PGE}_{2}$ (kindly provided by P. Isakson, Monsanto, Skokie, Illinois, USA; ref. 36) caused a block in the DN-to-DP transition (data not shown). Four different subtypes of $\mathrm{PGE}_{2}$ receptors (EP1-EP4) have been described in the mouse (15), and we detected all of them in the mouse embryonic thymus using RT-PCR (data not shown). Among the EP agonists, butaprost (an EP2 agonist) and 11deoxy $\mathrm{PGE}_{2}$ (an EP2/EP4 agonist) rescued the inhibition on DP cells (Figure 7a), whereas sulprostone (an EP1/EP3 agonist) and 17-phenyl-trinor PGE (an EP1 agonist) did not show any effect (data not shown). Therefore, the EP2 receptor subtype is most likely to mediate the effects of $\mathrm{PGE}_{2}$ on DP thymocyte formation.

We next attempted to rescue impaired CD4 SP maturation caused by selective COX-2 inhibitors in E17.5 FTOCs treated with $40 \mu \mathrm{M}$ of NS-398. $\mathrm{PGE}_{2}$ and 17phenyl trinor $\mathrm{PGE}_{2}$ (an EP1 agonist) dose dependently rescued the effect of the selective COX-2 inhibitor on CD4 SP formation, whereas 11-deoxy PGE 2 (an EP2/EP4 agonist) was ineffective (Figure $7 \mathrm{~b}$ ). Therefore, the EP1 receptor subtype is most likely to mediate the effects of $\mathrm{PGE}_{2}$ on CD4 SP thymocyte formation.

\section{Discussion}

To our knowledge, the present studies provide the first direct genetic and pharmacological evidence that both COX isozymes are required for the efficient maturation of the T-cell lineage in the fetal thymus. A model based on our data for the distinct function of the COX isozymes in fetal T-cell development is depicted in Figure 8.

Previous workers have noted the existence of PG synthases in the thymus $(14-18,37)$. We have extended these descriptive observations. Both COX isoforms are present in the fetal thymus. Furthermore, they differ in both the spatial and temporal aspects of their expression. Thus, COX-1, but not COX-2, is present in immature thymocytes, whereas COX-2 expression changes as the thymus matures. As a result, COX-2 is sparsely expressed in the fetal thymus, whereas it becomes highly expressed and localized to the medulla when the thymus is fully developed. In addition to varied patterns of expression, we report distinct functions for the 2 COXs in lymphocyte development. Spatial segregation of expression of these isozymes within a single organ is also observed in the kidney, where COX-1 is present in the collecting ducts, microvasculature, and glomeruli, and COX-2 is expressed in the macula densa and in a subset of medullary interstitial stroma cells $(1,2)$. Although distinct roles for the COX isozymes in renal function seem to be likely, these roles remain to be established.

Both genetic and pharmacological evidence from the present study supports a role for COX-1 in the transition 


\section{Figure 7}

Effect of PGE 2 and its analogues in inhibitor-treated FTOCs. (a) E15.5 FTOCs were treated with indomethacin $(40 \mu \mathrm{M})$ in the presence or absence of different concentrations of $\mathrm{PGE}_{2}$ or its synthetic analogues. After 5 days of culture, thymic lobes were dissociated, and the thymocytes were counted and stained for CD4 and CD8. DP counts are expressed in the graphs as the percentage of vehicle-treated FTOCs (100\%). (b) The same experiments were performed with E17.5 FTOCs in the presence of $40 \mu \mathrm{M}$ NS-398, and the CD4 SP cells were considered for the analyses. ${ }^{*} P<0.01$ vs. inhibitor-treated FTOCs.

of immature DN thymocytes to DP thymocytes. Furthermore, the presence of COX-1 in the most immature thymocytes, and its downregulation in the mature CD4 SP cells, also supports this role, although indirectly. The DN-to-DP transition depends upon productive TCR- $\beta$ gene rearrangements and the assembly of a pre-TCR complex $(38,39)$. However, because COX-1 inhibitors blocked the DN-to-DP transition following CD $3 \varepsilon$ crosslinking in $R A G-1^{-/-}$thymus, it is likely that the requirement for PG synthesis follows signaling by the pre-TCR. Furthermore, COX-1 inhibitors did not block the antiCD3E-induced transition of $R A G-1^{-/-}$thymocytes to the intermediate CD8 SP subset, although they reduced the level of expression of CD8 on this subset (data not shown). Interestingly, overexpression of the transcription factor Egr-1 induces T-cell development in $R A G-1^{-/}$ mice to the immature CD8 SP stage, but the cells remain blocked prior to CD4 upregulation (40). Presently, it is unknown whether or how the COX-1 and Egr-1 pathways might interact to control this step of T-cell differentiation. Our experiments support a role for COX-1 in the transition of immature DN thymocytes to DP thymocytes. Although it remains to be determined whether COX-1 is required for survival, proliferation, or differentiation of thymocytes, COX-1 inhibition does not cause a general increase in thymocyte apoptosis. Other

\footnotetext{
Figure 8

Simplified model of the possible role of COX-1, COX-2, PGs, and PG receptors during T-cell development. COX1-dependent $\mathrm{PGE}_{2}$ synthesis is required for an efficient transition of thymocytes from DN to DP. In this model, an autocrine effect of $P G E_{2}$ acting on the EP2 receptor of immature thymocytes is hypothesized. However, we cannot exclude an effect of COX-1 in stromal cells. COX-2-dependent PG production positively affects the DN population, and it is also required for CD4 SP formation. We hypothesize a paracrine effect of $\mathrm{PGE}_{2}$ formed by stromal cell COX-2 acting on thymocyte EP1 receptors in positive selection. It is also possible that COX-2-dependent PG production may also act on the stromal cells in an autocrine fashion.
}

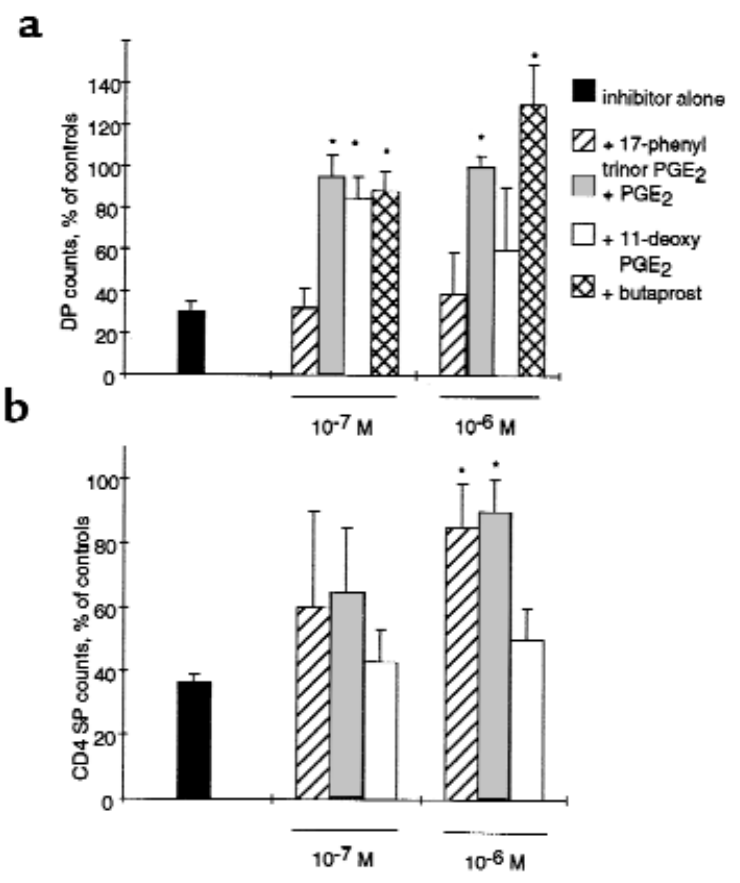

groups reported conflicting effects on apoptosis by PGs, either protecting (41) or inducing (42) programmed cell death. This conflict may reflect the different experimental conditions used by other investigators. In fact, these previous reports studied isolated thymocytes incubated with PGs for relatively short periods. FTOCs, on the other hand, represent a more integrated environment where the anatomy of the thymus is preserved. Therefore, an isolated cell system may fail to reflect the interactions operating in the intact whole organ.

Selective COX-1 inhibition did not affect the positive selection of CD4 SP lymphocytes. However, there was an increased percentage and increased absolute numbers of the CD4 SP cells in COX-1 $1^{-/-}$thymi. Expression of COX2 was increased in $\mathrm{COX}^{-11^{-/}}$thymi. Compensatory expression of the alternate isozyme has been reported in COX-deficient lung fibroblasts (43), whereas compensatory expression was not observed in colonic adenomes of patients treated with a COX-2 inhibitor (DuBois, R., personal communication). In the thymus, it is likely that the COX-2-dependent PG production may partially compensate for the absence of COX-1. Indeed, the block in transition to DP cells is more complete in the presence of inhibitors (Figure 5b) than following gene deletion (Figure 2a). COX-2 overexpression may also cause the increase in formation of CD4 SP cells in $\mathrm{COX}-1^{-/-}$thymi.

Both mutation of the COX-2 gene and pharmacological inhibition of the enzyme limited the fetal development of CD4 SP thymocytes. Moreover, COX-2 protein was upregulated in $\mathrm{COX}-1^{-/-}$thymi, coinciding with a significant increase of CD4 SP thymocytes. Inhibition of COX-2 failed to affect negative selection by deletion (our unpub- 
lished data). Thus, COX-2-dependent PG synthesis appears to influence CD4 SP differentiation. Although the existence of such a system has previously been hypothesized $(44,45)$, PGs would represent the first family of mediators, aside from the TCR, its coreceptors, and their ligands, that modulates the CD4 SP development in intact thymic organs. As COX-2 is only expressed in stromal cells, it seems likely that PGs act as stromal cofactors to influence developing thymocytes (Figure 8). Indeed, we have shown that COX-2-dependent formation of PGs enhanced adherence of DP and CD4 SP thymocytes to medullary stromal cell lines (29). Because we saw no effect of COX-2 deficiency on CD8 generation, it is possible that COX-2 may function specifically in the CD4 lineage.

Genetic deletion or inhibition of COX-2 negatively influenced the DN cells, especially the CD44-CD $25^{+}$subpopulation, which represents the majority (>70\%) of DN thymocytes (35). Subsequent proliferation may amplify the progenitors that slip past the $\mathrm{CD} 44^{-} \mathrm{CD} 25^{+}$stage. CD25 upregulation coincides with the initiation of TCR$\beta$ rearrangement, and CD 44 downregulation is associated with the acquisition of TCR- $\beta$ cytoplasmic expression (46). Therefore, it is possible that COX- 2 may be required in some aspect of the initiation or progression of TCR- $\beta$ rearrangements.

Finally, our data support a predominant role for $\mathrm{PGE}_{2}$ in mediating both the effect of COX-1 during DN-to-DP transition and the effect of COX-2 in CD4 SP formation, although through 2 distinct EP subtypes: EP2 and EP1, respectively. Both are $G$ protein-coupled receptors: EP2 activates adenylyl cyclase, and EP1 is coupled to phospholipase $C$ (15). A potential mechanism by which $\mathrm{PGE}_{2}$ may act through the EP2 subtype is the modulation of cellular adhesion, because cAMP analogues and $\mathrm{PGE}_{2}$ both enhance the binding of immature DN and DP thymocytes to extracellular matrix proteins (47). Alternatively, a transient elevation of cAMP is necessary for the initiation of $S$ phase in mouse lymphocytes (48), suggesting that $\mathrm{PGE}_{2}$, acting through EP2, may modulate cellular proliferation.

In summary, we report evidence that COX isozymes play functionally distinct roles in lymphocyte development, in addition to their recognized importance in the regulation of inflammation and of mature $\mathrm{B}$ - and $\mathrm{T}$ helper cells. COX-1-dependent formation of $\mathrm{PGE}_{2}$, acting through EP2 receptors, plays an important role in DN-to-DP transition, while COX-2 expression in the stroma influences positive selection of thymocytes through EP1 receptors.

Immunomodulatory effects of nonsteroidal antiinflammatory drugs in the induction and maintenance of central tolerance have been reported previously (49-51). Interestingly, indomethacin prevents induction of thymic tolerance in vivo in mice, while administration of $\mathrm{PGE}_{2}$ rescues this effect (49). Although an analysis of immune function has yet to be reported receptors or in mice deficient in either COX isoform or in prostanoid receptors, idiopathic chronic suppurative peritonitis and bowel inflammation were both unexpected findings in the COX-2 knockout mice (21). Given the emergence of selective inhibitors of COX isozymes into clinical application, assessment of their effects on immune function and immune cell development would seem timely.

\section{Acknowledgments}

Supported by grants HL-57847 and HL-54500 from the National Institutes of Health.

1. Smith, W.L., and DeWitt, D.L. 1996. Prostaglandin endoperoxide H synthases -1 and -2. Adv. Immunol. 62:167-215.

2. Herschman, H.R., Reddy, S.T., and Xie, W. 1997. Function and regulation of prostaglandin synthase-2. Adv. Exp. Med. Biol. 407:61-66.

3. Betz, M., and Fox, B.S. 1991. Prostaglandin $E_{2}$ inhibits production of Th1 lymphokines but not of Th2 lymphokines. J. Immunol. 146:108-113.

4. Anastassiou, E.D., Paliogianni, F., Balow, J.P., Yamada, H., and Boumpas, D.T. 1992. Prostaglandin $E_{2}$ and other cyclic AMP-elevating agents modulate IL-2 and IL-2R alpha gene expression at multiple levels. J. Immunol. 148:2845-2852.

5. Paliogianni, F., Kincaid, R.L., and Boumpas, D.T. 1993. Prostaglandin $E_{2}$ and other cyclic AMP elevating agents inhibit interleukin 2 gene transcription by counteracting calcineurin-dependent pathways. J. Exp. Med. 178:1813-1817.

6. Hilkens, C.M.U., et al. 1995. Differential modulation of T helper type 1 (Th1) and T helper type 2 (Th2) cytokine secretion by prostaglandin $\mathrm{E}_{2}$ critically depends on interleukin 2. Eur. J. Immunol. 25:59-63.

7. Katamura K., et al. 1995. Prostaglandin $\mathrm{E}_{2}$, at priming doses of naive $\mathrm{CD}^{+}$T-cells inhibits acquisition of ability to produce IFN- $\gamma$ and IL-2, but not IL-4 and IL-5. J. Immunol. 155:4604-4612.

8. Van der Pouw Kraan, T.C.T.M., Boeije, L.C.M., Smeenk, R.J.T., Wijdenes, J., and Aarden, L.A. 1995. Prostaglandin $\mathrm{E}_{2}$ is a potent inhibitor of human interleukin 12 production. J. Exp. Med. 181:775-779.

9. Fedyk, E.R., and Phipps, R.P. 1996. Prostaglandin $E_{2}$ receptors of the EP2 and EP4 subtypes regulate activation and differentiation of mouse B lymphocytes to IgE-secreting cells. Proc. Natl. Acad. Sci. USA. 93:10978-10983.

10. Mastino, A., et al. 1993. Correlation between induction of lymphocyte apoptosis and prostaglandin $\mathrm{E}_{2}$ production by macrophages infected with HIV. Cell. Immunol. 152:120-130.

11. Delemarre, F.G., et al. 1995. Reduced toxoplasmatic activity of monocytes and monocyte-derived macrophage from AIDS patients is mediated via prostaglandin $\mathrm{E}_{2}$. AIDS. 9:441-445.

12. Cayeux, S.J., Beverly, P.C., Schulz, R., and Dorken, B. 1993. Elevated plasma prostaglandin $\mathrm{E}_{2}$ levels found in 14 patients undergoing autologous bone marrow or stem cell transplantation. Bone Marrow Transplant. 12:603-608.

13. Leung, D.Y. 1995. Atopic dermatitis: the skin as a window into the pathogenesis of chronic allergic diseases. J. Allergy Clin. Immunol. 96:302-318.

14. Nusing, R., et al. 1992. Localization of thromboxane synthase in human tissues by monoclonal antibody Tu 300. Virchows Arch. A Pathol. Anat. Histopathol. 421:249-254.

15. Ushikubi, F., Hirata, M., and Narumiya, S. 1995. Molecular biology of prostanoid receptors: an overview. J. Lipid Mediat. Cell Signal. 12:343-359.

16. Appasamy, P.M., et al. 1991. Expression of prostaglandin G/H synthase (cyclooxygenase) during murine fetal thymic development. Cell. Immunol. 137:341-357.

17. Milicevic, N.M., Appasamy, P.M., Colic, M., and Milicevic, Z. 1994. Immunocytochemical demonstration of prostaglandin synthase (cyclooxygenase) in thymic macrophages of normal and cyclosporintreated rats. Immunobiology. 190:376-384.

18. Sun, L., Piltch, A.S., Liu, P., Johnson, A., and Hayashi, J. 1990. Thymocytes stimulate metabolism of arachidonic acid in rat epithelial cells. Cell. Immunol. 131:86-97.

19. McCormack, J.E., Kappler, J., Marrack, P., and Westcott, J.Y. 1991. Production of prostaglandin E2 and prostacyclin by thymic nurse cells in culture. J. Immunol. 146:239-243.

20. Pendino, K.J., Chepenik, K.P., and Schmidt, R.R. 1992. Differential eicosanoid synthesis by murine fetal thymic non-lymphoid cells. Immunol. Cell Biol. 70:237-252.

21. Morham, S.F., et al. 1995. Prostaglandin synthase gene disruption causes severe renal pathology in the mouse. Cell. 83:473-482.

22. Langenbach, R., et al. 1995. Prostaglandin synthase-1 gene disruption in mice reduce arachidonic acid-induced inflammation and indomethacininduced gastric ulceration. Cell. 83:483-492.

23. Kisielow, P. 1990. Applications of fetal thymus organ cultures in studies of T-cell development. In Immunological methods, volume 4. I. Lefkovits and B. Pernis, editors. Academic Press. San Diego, CA. 292-309.

24. Arakawa, T., et al. 1996. Prostanoid receptors of murine NIH 3 T3 and RAW 264.7 cells. Structure and expression of the murine prostaglandin EP4 receptor gene. J. Biol. Chem. 271:29569-29575.

25. Fedyk, E.R., Ripper, J.M., Brown, D.M., and Phipps, R.P. 1996. A molecular analysis of the PGE receptor (EP) expression on normal and transformed B lymphocytes: coexpression of the EP1, EP2, EP3, and EP4. Mol. Immunol. 33:33-45.

26. Habib, A., et al. 1993. Demonstration of an inducible cyclooxygenase in 
human endothelial cells using antibodies raised against the carboxylterminal region of the cyclooxygenase-2.J Biol Chem. 268:23448-23454.

27. Jenkinson, E.J., and Owen, J.J. 1990. T-cell differentiation in thymus organ cultures. Semin. Immunol. 2:51-58.

28. Bhattacharyya, D.K., Lecomte, M., Dunn, J., Morgans, D.J., and Smith, W.L. 1995. Selective inhibition of prostaglandin endoperoxide synthase-1 (cyclooxygenase-1) by valeryl salicylic acid. Arch. Biochem. Biophys. 317:19-24.

29. Rocca, B., Spain, L.M., Ciabattoni, G., Patrono, C. and FitzGerald, G.A. 1999. Differential expression and regulation of cyclooxygenase enzymes in thymic stromal cells. J. Immunol. 162:4589-4597.

30. Patrignani, P., et al. 1994. Biochemical and pharmacological characterization of the cyclooxygenase activity of human blood prostaglandin endoperoxide synthases. J. Pharmacol. Exp. Ther. 271:1705-1712.

31. Smith, W.L., and DeWitt, D.L. 1995. Biochemistry of prostaglandin endoperoxide $H$ synthase-1 and synthase -2 and their differential susceptibility to nonsteroidal anti-inflammatory drugs. Semin. Nephrol. 15:179-194.

32. Mombaerts, P., et al. 1992. RAG-1 deficient mice have no mature B and T-lymphocytes. Cell. 68:869-877.

33. Levelt, C.N., Mombaerts, P., Iglesias, A., Tonegawa, S. and Eichmann, K. 1993. Restoration of early thymocyte differentiation in T-cell receptor $\beta$-chain deficient mutant mice by transmembrane signaling through CD3E. Proc. Natl. Acad. Sci. USA. 90:11410-11415.

34. Panara, M.R., et al. 1995. Effects of the novel anti-inflammatory compounds, $\mathrm{N}$-[2-(cyclohexyloxy)-4-nitrophenyl] merthanesulphonamide (NS-398) and 5-methane-sulphonamido-6-(2,4-difluorothio-phenyl)-1 indanone (L-745,337), on the cyclooxygenase activity of human blood prostaglandin endoperoxide synthases. Br. J. Pharmacol. 116:2429-2434.

35. Godfrey, D.I., Kennedy, J., Suda, T., and Zlotnik, A. 1993. A developmental pathway involving four phenotypically and functionally distinct subsets of CD3-DN triple negative adult mouse thymocytes defined by CD44 and CD25 expression. J. Immunol. 150:4244-4252.

36. Mnich, S.J., et al. 1995. Characterization of a monoclonal antibody that neutralizes the activity of prostaglandin $\mathrm{E}_{2}$.J. Immunol. 155:4437-4444.

37. O'Neill, G.P., and Ford-Hutchinson, A.W. 1993. Expression of mRNA for cyclooxygenase- 1 and cyclooxygenase- 2 in human tissues. FEBS Lett. 330:156-160.

38. Saint-Ruf, C., et al. 1994. Analysis and expression of a cloned pre-T-cell receptor gene. Science. 266:1208-1212.

39. Berger, M.A., et al. 1997. Subunit composition of pre-T-cell receptor com- plexes expressed by primary thymocytes: $C D 3 \varepsilon$ is physically associated but not functionally required. J. Exp. Med. 186:1461-1467.

40. Miyazaki, T. 1997. Two distinct steps during thymocyte maturation from CD4-CD8- to CD4+CD8+ distinguished in the early growth response (Egr)-1 transgenic mice with a recombinase-activating genedeficient background. J. Exp. Med. 186:877-885.

41. Goetzl, E.J., Songzhu, A., and Zeng, L. 1995. Specific suppression by prostaglandin $\mathrm{E}_{2}$ of activation-induced apoptosis of human DP T lymphoblasts. J. Immunol. 154:1041-1047.

42. Saiagh, S., Rigal, D., and Monier, J.C. 1994. Effects of $\mathrm{PGE}_{2}$ upon differentiation and programmed cell death of suspension cultured CD4-CD8- thymocytes. Int. J. Immunopharmacol. 16:775-786.

43. Kirtikara, K., et al. 1998. Compensatory prostaglandin E2 biosynthesis in cyclooxygenase 1 or 2 null cells. J. Exp. Med. 187:517-523.

44. Anderson, G., Owen, J., Moore, N.C., and Jenkinson, E.J. 1994. Thymic epithelial cells provide unique signals for positive selection of $\mathrm{CD} 4{ }^{+} \mathrm{CD}^{+}$ thymocytes in vitro. J. Exp. Med. 179:2027-2031.

45. Pénit, C., Lucas, B., and Vasseur, F. 1995. Cell expansion and growth arrest phase from precursor (CD4-8-) to immature (CD4+8+) thymocytes in normal and genetically modified mice. J. Immunol. 154:5103-5113.

46. Falk, I., Biro, J., Kohler, H., and Eichmann, K. 1996. Proliferation kinetics associated with $\mathrm{T}$-cell receptor-beta chain selection of fetal murine thymocytes. J. Exp. Med. 184:2327-2339.

47. Halvorson, MJ., and Coligan, J.E. 1995. Enhancement of VLA integrin receptor function on thymocytes by cAMP is dependent on the maturation stage of the thymocytes. J. Immunol. 155:4567-4574.

48. Wang, T., Sheppard, J.R, and Foker, J.E. 1978. Rise and fall of cyclic AMP is required for onset of lymphocyte A synthesis. Science. 211:155-157.

49. Scheuer, W.V., Hobbs, M.V., and Weigle, W.O. 1987. Interference with tolerance induction in vivo by inhibitors of prostaglandin synthesis. Cell. Immunol. 104:409-418.

50. Ruiz, P., Woodson, C., and Rey, L. 1992. Modification of rat skin allograft rejection by inhibition of thromboxane synthesis. Transplantation. 54:385-387.

51. Remuzzi, G., et al. 1994. Thromboxane A2 receptor blocking abrogates donor specific unresponsiveness to renal allografts induced by thymic recognition of major histocompatibility allopeptides. J. Exp. Med. 180:1967-1972 